\title{
Lance-Adams Syndrome
}

\author{
Jun-Hwa Shin, M.D., Jong Moon Park, M.D., A Ram Kim, M.D., Hee Suk Shin, M.D., \\ Eun Shin Lee, M.D., Min-Kyun Oh, M.D., Chul Ho Yoon, M.D.
}

\begin{abstract}
Department of Rehabilitation Medicine and Institute of Health Sciences, Gyeongsang National University
\end{abstract} Graduate School of Medicine, Jinju 660-702, Korea

It is not common for a patient who survives cardiac arrest to experience significant neurologic impairment such as acute and chronic post-hypoxic myoclonus, known as Lance-Adams syndrome. This syndrome is predominantly characterized by myoclonus that starts days to weeks after cardiopulmonary resuscitation in patients who regained consciousness. Although several cases of LAS were reported, the decisive treatment method has not been established. We report a 43 year old man with Lance-Adams syndrome who showed long-term improvement through treatment with anti-myoclonic agents and participation in a rehabilitation program.

Key Words Cardiopulmonary resuscitation, Myoclonus, Lance-Adams syndrome

\section{INTRODUCTION}

Through the recent development of medical technologies, the number of patients who survive a cardiac arrest is increasing. However, such patients tend to have diverse and severe sequelae. In rare cases, they may suffer from dyskinesia resulting from hypoxic brain damageand their disorders include Parkinson's disease, dystonia, chorea, athetosis, tremor, and Lance-Adams syndrome (LAS). ${ }^{1}$

Post-hypoxic myoclonus (PHM) refers to myoclonus occurring after hypoxic brain damage resulting from a cardiac arrest, with abrupt and irregular contractions of muscles as if they were jumping lightly. ${ }^{2}$ This disease, ac-

Received May 19, 2011; Accepted October 13, 2011

Corresponding author: Chul Ho Yoon

Department of Rehabilitation Medicine and Institute of Health Sciences, Gyeongsang National University Graduate School of Medicine, 90, Chilam-dong, Jinju 660-702, Korea

Tel: +82-55-750-8255, Fax: +82-55-750-8255, E-mail: yoonch@gnu.ac.kr

(ㄷ) This is an open-access article distributed under the terms of the Creative Commons Attribution Non-Commercial License (http:// creativecommons.org/licenses/by-nc/3.0) which permits unrestricted noncommercial use, distribution, and reproduction in any medium, provided the original work is properly cited.

Copyright $\odot 2012$ by Korean Academy of Rehabilitation Medicine cording to its onset time, may be divided into two types, acute and chronic. Acute PHM is also called post-hypoxic myoclonic status epilepticus and refers to myoclonus in a patient with a coma that occurs within 48 hours after a cardiac arrest. Chronic PHM, also known as LanceAdams Syndrome, refers to myoclonus that starts days to weeks after cardiopulmonary resuscitation in patients who regained consciousness. ${ }^{1,2}$ According to Werhahn et al., ${ }^{3}$ LAS patients show varying problems in cognitive function from normal to severe dysfunction.

LAS was first reported by Lance and Adams ${ }^{4}$ in 1963 after he observed muscle cramps characteristic of patients who survived cardiac arrest. As for domestic studies, Choi and Song. ${ }^{2}$ examined whether treatment was necessary in 82 acute PHM patients and Kim et al. ${ }^{5}$ reported a case in which a 15-month-old infant hospitalized for croup developed LAS. There are reports on acute and chronic PHM patients in Korea as well but there are few reported cases of adult patients with LAS.

Accordingly, the authors report this case because we have experienced an adult patient with chronic PHM, in other words LAS, which occurred days after a cardiac arrest. 


\section{CASE REPORT}

A 43-year-old man was brought to the emergency room due to bilateral shoulder and back pain after he fell off a three-story building. When he arrived at the emergency room, there were no other specific findings in tests for cognitive and neurological conditions and in a blood test. However, four hours after his arrival, he had an abrupt cardiac arrest with no obvious cause and cardiopulmonary resuscitation (CPR) was performed for about 3 minutes. All his vital signs were back to normal after the CPR but he was in a coma for about 30 minutes during which generalized tonic clonic seizures continued with the whole body stiff and trembling. He stayed in the neurology department for around 1 month to treat his status epilepticus and agents like midazolam, propofol, and sodium valproate were administered. As the onemonth treatment period ended, he gradually recovered consciousness and the symptoms of continuous seizures during the acute phase were alleviated. Instead, seizures, which are contractions of muscles as if lightly jumping during sputum suction through the tracheostomy tube or pain stimulus, newly emerged. The amount of medications intravenously injected to treat the status epilepticus was gradually reduced and sodium valproate, levetiracetam, and clonazepam were orally administered in order

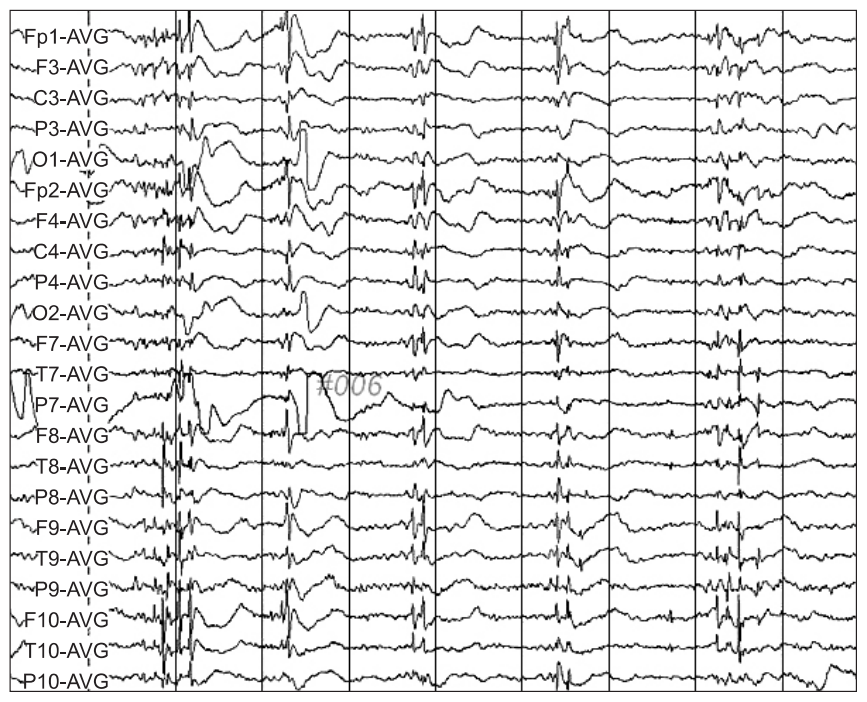

Fig. 1. One day after cardiopulmonary resuscitation (CPR), the electroencephalogram (EEG) of the patient showed semiperiodic bursts of spike-and-waves and polyspike-and-wave-complexes, maximally in the frontal area. to reduce the new patterns of seizures. For rehabilitation, the patient was moved to the department of rehabilitation medicine because he had problems with gait, language use, and activities of daily living (ADL) resulting from myoclonus.

When he moved to the rehabilitation department, he had normal muscle strength in the upper and lower extremities and the neck, but he had myoclonus with irregular contractions of muscles as if jumping lightly in the upper and lower extremities, trunk, face, and tongue. Such muscle cramps were aggravated during emotional changes or voluntary actions and decreased while he was resting or sleeping. He scored 3 in the mini-mental state examination (MMSE), which indicated severe cognitive disabilities. He was entirely dependent on the assistance of others for functional activities such as sitting on the bed, walking, performing simple manual movements , and eating. He needed a wheelchair in order to move.

One day after the CPR, when he had continuous symptoms of muscle cramps, his electroencephalogram (EEG) showed cyclic epileptiform waves (Fig. 1). However, after his move to the rehabilitation department, the epileptiform waves did not appear in the EEG when his muscle cramps had been stabilized (Fig. 2). EEG was performed when his condition did not change except for aggravated myoclonus after the dose of clonazepam was reduced. At this time, the epileptiform waves did not appear and only waveforms suggesting hypoxic brain injury were observed (Fig. 3).

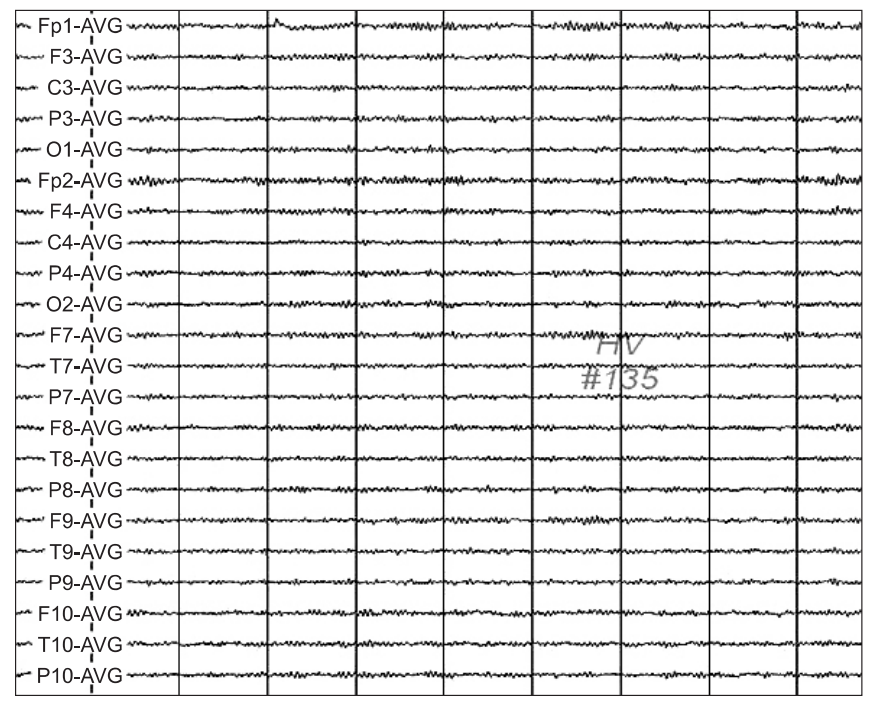

Fig. 2. One month after CPR, the EEG showed no epileptiform activities. 
Brain magnetic resonance imaging performed three months after the CPR showed a slightlyincreased intensity of signals spreading over the white matter of both hemispheres, suggesting hypoxic encephalopathy (Fig. 4).

For about 4 months after he moved to the rehabilitation department, levetiracetam $200 \mathrm{mg}$, sodium valproate $1,800 \mathrm{mg}$, and clonazepam $4.5 \mathrm{mg}$ were administered and occasional myoclonus gradually improved. During physiotherapy, there were symptoms of weakness, considered to be because of anticonvulsants, and a decision to reduce the amount of medications was made. We referred to a recent study by Frucht et al. ${ }^{6}$ stating that levetiracetam was effective in LAS and reduced the amount of clonazepam and observed aggravation of myoclonus.

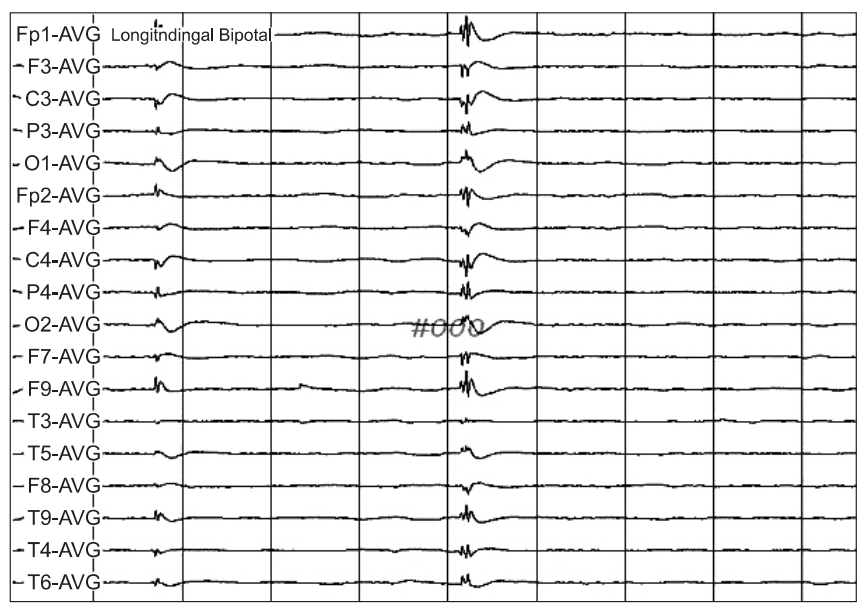

Fig. 3. Three months after CPR, the EEG showed low voltage background activity though the artifacts at the multiple electrode.
Therefore, we increased the dose of clonazepam to the prior level and the myoclonus improved to its original state. Due to the patient's sustained weakness, we reduced the amount of levetiracetam, but the weakness did not improve. However, a finding of aggravated myoclonus was not observed as it was when the amount of clonazepam was decreased.

During hospitalization, he received progressive rehabilitation treatment as well as drug treatment. He had a lot of difficulty in gait and ADL because of myoclonus during voluntary actions, which was accompanied by dysphagia resulting from involuntary spasms of facial and tongue muscles. To resolve this problem, intensive gait, ADL, and swallowing therapy was performed. In particular, slow movements were induced during gait and ADL therapy because they led to a relatively less myoclonus.

He was discharged about 8 months after the CPR; he was able to sit on the bed, grab a string and stand for around 15 minutes with minimal assistance. His condition improved so he could move by himself in a wheelchair although independent walking was impossible. His cognitive function improved as well he scored 21 in the MMSE.

\section{DISCUSSION}

LAS is characterized by myoclonus that starts days to weeks after a cardiac arrest. The myoclonus is initiated by movement, startling, and tactile stimuli and alleviated when a patient rests or sleeps. ${ }^{3,4,7}$ The patient may be diagnosed with LAS because he has clinical myoclonus
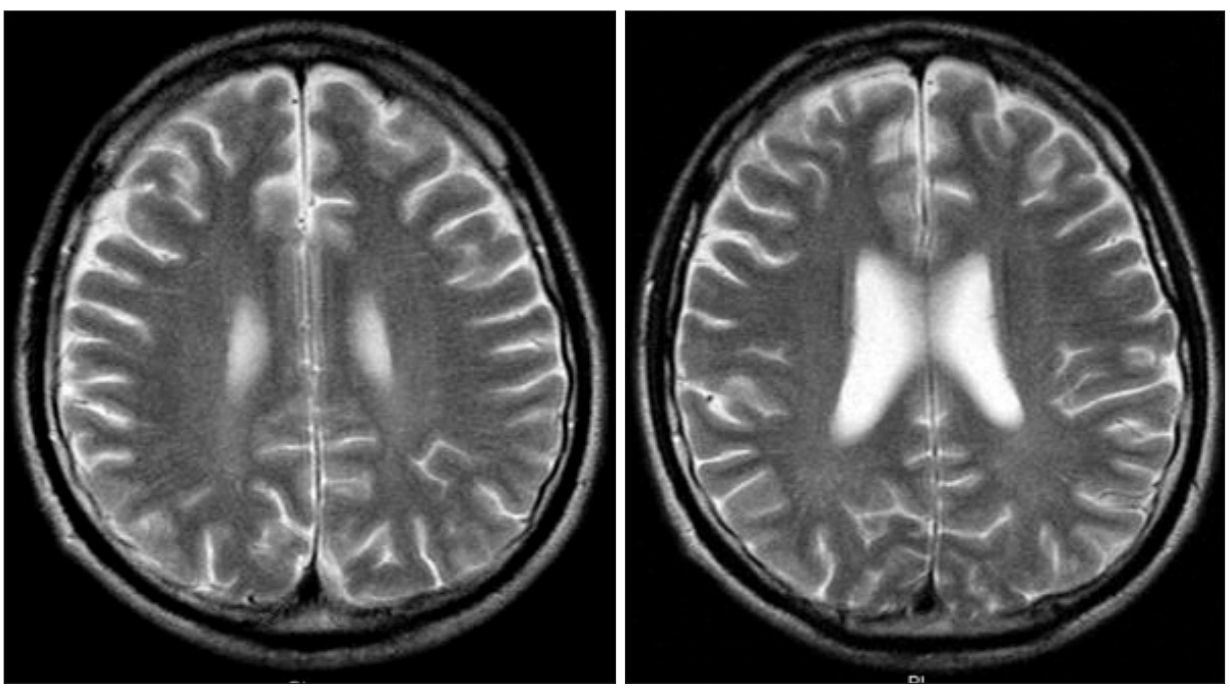

Fig. 4. Three months after CPR, T2-weighted magnetic resonance axial images of the patient showed subtle hyperintense lesions in the both cerebral white matters, suggesting hypoxic encephalopathy. 
characteristic of the patients reported by Lance and Adams. ${ }^{4}$ It is known that the pathophysiology of LAS is related to an abnormal finding of diverse neurochemicals, in particular, a loss of serotonin (5-hydroxytryptamine or 5-HT), which 5-Hydroxytryptophan (5-HTP) is decarboxylated ${ }^{3}$, gamma-amino butyric acid (GABA).

Substances like GABA affect the clinical progression of LAS by influencing the 5 -HT system. ${ }^{8}$

However, the exact pathophysiologyof LAS is still not clear and therefore there is no guideline for drug treatment of the disease. As a result, treatment is limited and is often decided on empirically. Frucht and Fahn. ${ }^{9}$ reported that about $50 \%$ of the LAS patients experienced improvement in their symptoms after clonazepam, sodium valproate, and piracetam were administered. A recent study reported that levetiracetam was effective in regulating the symptoms of LAS. ${ }^{6}$ Based on a number of studies, the authors decided that clonazepam, sodium valproate, piracetam, and levetiracetam were good primary drugs to treat LAS and verified that the combined administration of sodium valproate, clonazepam, and levetiracetam to the patient of this case was effective in his myoclonus. Nonetheless, the dose of clonazepam affected myoclonus more than the dose of levetiracetam, which was shown to be an effective agent by a recent study the authors reached the conclusion that clonazepam was more effective in reducing myoclonus than levetiracetam. In this case, the patient's myoclonus pattern was affected more significantly when adjusting the dose of clonazepam than when adjusting the dose of levetiracetam. Therefore, more caution is necessary when adjusting the dose of clonazepam for an LAS patient.

Werhahn et al. ${ }^{3}$ reported that the prognosis was good when the treatment started in early stage LAS. Meanwhile, Polesin and Stern. ${ }^{10}$ noted that gait and ADL therapy together with drug treatment slowed the progression of LAS and prevented additional disabilities. The precise cause of LAS is not known. Prognosis may be good when medications are appropriately administered and rehabilitation is done at an early stage of the disease. When a patient who regains consciousness after CPR has symptoms of myoclonus and is suspected of having LAS, (i) aggressive drug treatment to reduce the myoclonus and (ii) rehabilitation to prevent disabilities are necessary. Additional research to examine the cause of the disease and aimed at discovering new treatment methods should be done.

\section{REFERENCES}

1. Venkatesan A, Frucht S. Movement disorders after resuscitation from cardiac arrest. Neurol Clin 2006; 24: 123-132

2. Choi HC, Song HK. Is the intensive anticonvulsant treatment for control of acute posthypoxic myoclonic status epilepticus necessary? J Korean Neurol Assoc 2006; 24: 125-130

3. Werhahn KJ, Brown P, Thompson PD, Marsden CD. The clinical features and prognosis of chronic posthypoxic myoclonus. Mov Disord 1997; 12: 216-220

4. Lance JW, Adams RD. The syndrome of intention or action myoclonus as a sequel to hypoxic encephalopathy. Brain 1963; 86: 111-136

5. Kim DH, Kim SJ, Oh KI, Kwon YS, Son BK. A Case of the successful treatment of pentobarbital for posthypoxic action myoclonus (Lance-Adams Syndrome) with refractory status epilepticus. J Korean Child Neurol Soc 2006; 14: 342-347

6. Frucht SJ, Louis ED, Chuang C, Fahn S. A pilot tolerability and efficacy study of levetiracetam in patients with chronic myoclonus. Neurology 2001; 57: 11121114

7. Welsh JP, Placantonakis DG, Warsetsky SI, Marquez RG, Bernstein L, Aicher SA. The serotonin hypothesis of myoclonus from the perspective of neuronal rhythmicity. Adv Neurol 2002; 89: 307-329

8. Matsumoto RR, Truong DD, Nguyen KD, Dang AT, Hoang TT, Vo PQ, Sandroni P. Involvement of GABA(A) receptors in myoclonus. Mov Disord 2000; 15: 47-52

9. Frucht S, Fahn S. The clinical spectrum of posthypoxic myoclonus. Mov Disord 2000; 15: 2-7

10. Polesin A, Stern M. Post-anoxic myoclonus: a case presentation and review of management in the rehabilitation setting. Brain Inj 2006; 20: 213-217 